\title{
Laparoscopic Management of Renal Paratransplant Hernia
}

\author{
Duncan Yoder, Reza Ghasemian \\ Medstar Georgetown Transplant Institute, Washington DC, USA \\ Email: duncanpaulyoder@gmail.com
}

Received 29 December 2014; accepted 16 January 2015; published 22 January 2015

Copyright $@ 2015$ by authors and Scientific Research Publishing Inc.

This work is licensed under the Creative Commons Attribution International License (CC BY). http://creativecommons.org/licenses/by/4.0/

(c) (i) Open Access

\begin{abstract}
A renal paratransplant hernia is an unusual and potentially serious variant of an internal hernia. It is caused by entrapment of bowel through a defect in the peritoneum adjacent to the transplanted kidney. Abdominal pain and vomiting with or without distention are the main symptoms. Prompt diagnosis and early surgical intervention are critical to prevent serious morbidity and even mortality. Historically, renal paratransplant hernias were repaired via an exploratory laparotomy. We present our experience in two patients with renal paratransplant hernia who were treated with laparoscopic repair.
\end{abstract}

\section{Keywords}

\section{Renal Transplant, Hernia, Paratransplant Hernia, Laparoscopic Repair}

\section{Introduction}

Kyriakides et al. first reported a paratransplant hernia associated with kidney transplant as an entrapment of bowel through a defect in peritoneum overlying the transplanted kidney [1].

There have been nine cases of renal paratransplant hernias reported in the Pubmed literature. All of these cases were treated with exploratory laparotomy. Three cases required bowel resection and one case resulted in death [2].

Herein we report our experience with 2 cases of renal paratransplant hernias. Both cases were successfully managed laparoscopically thereby avoiding the morbidity associated with exploratory laparotomy. In one case, the defect involved peritoneum and an abdominal fascia layer.

\section{Case 1}

The patient is a 65-year-old African American male with a past medical history of end stage renal disease sec-

How to cite this paper: Yoder, D. and Ghasemian, R. (2015) Laparoscopic Management of Renal Paratransplant Hernia. International Journal of Clinical Medicine, 6, 60-63. http://dx.doi.org/10.4236/ijcm.2015.61009 
ondary to diabetes mellitus and hypertension.

He received a cadaveric kidney transplant. The allograft was placed in the left lower quadrant via a standard Gibson incision. Wound closure included the internal oblique muscle and trasversalis fascia in one layer followed by external oblique as a separate layer using nonabsorbable No. 0 suture material. Immunosuppression included Alemtuzumab induction followed by Mycophenolate and Tracolimus. Prednisone was not used.

The allograft functioned immediately and he was discharged on postoperative day five with a serum creatinine of $1.8 \mathrm{mg} / \mathrm{dL}$.

Four weeks post operatively he presented with pain over the incision, nausea, vomiting and obstipation. Examination of abdomen revealed mild distention with a localized prominence and tenderness over incision area. An abdominal CT scan showed dehiscence of internal oblique and tranversalis with an intact external oblique fascia. Small bowel was incarcerated between the layers (Figure 1).

An abdominal laparoscopy confirmed herniation of a loop of small bowel through the incision (Figure 2).

The hernia defect was inspected after reducing the small bowel back into the peritoneal cavity and revealed a dehiscence of the internal and transversalis layers. The external oblique remained intact. Essentially, the small bowel had herniated through the weaker deep transversalis and internal oblique fascia without disrupting the external oblique fascia which is the strength layer of the closure. Thus it had become trapped between the external oblique and internal oblique. The herniated segment of bowel appeared viable with normal peristalsis. The hernia defect was then widely incised to prevent future incarceration (Figure 3). The external layer appeared sufficient to support the abdominal cavity.

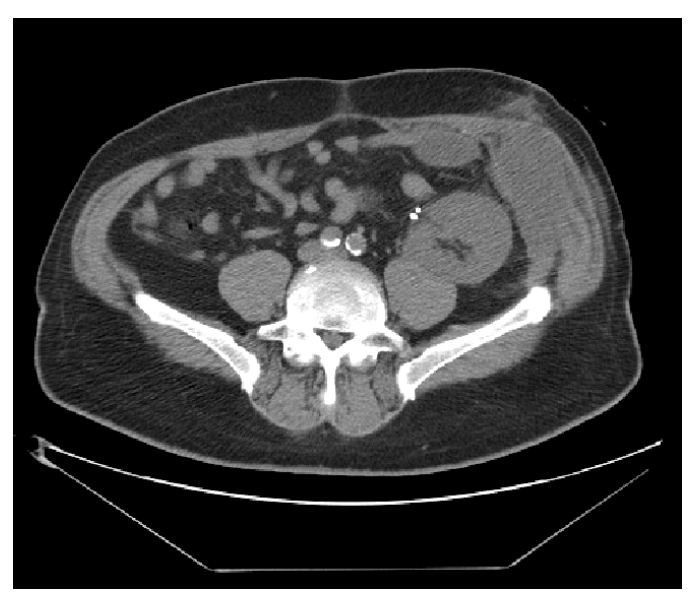

Figure 1. Abdominal Ct scan showing separation of internal oblique and transversalis fascia and intact external oblique with herniated loop of bowel.

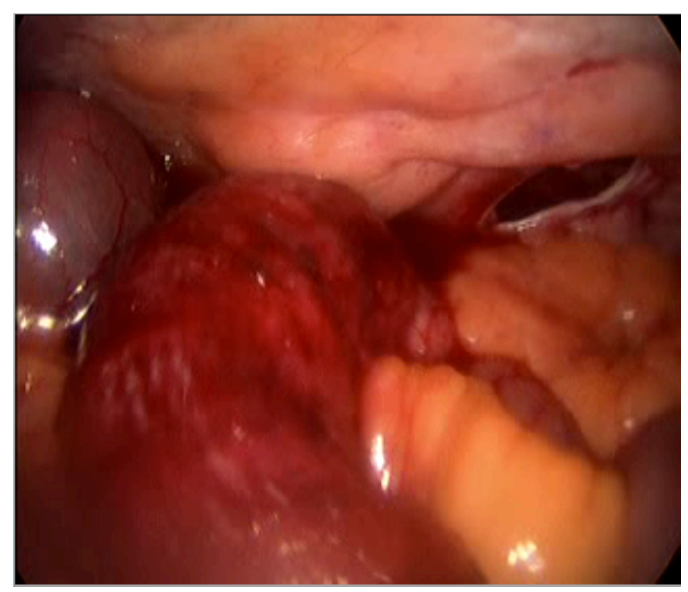

Figure 2. Laparoscopic view of the defect (blue arrow) and reduced loop of small bowel (white arrow). 


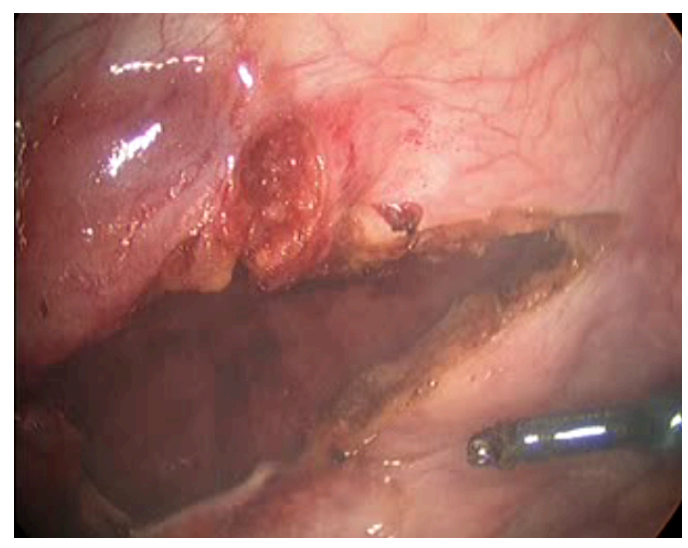

Figure 3. Laparoscopic view of the defect following incision.

The patient recovered uneventfully and has not experienced a recurrence to date. There is no asymmetry of the abdominal wall.

\section{Case 2}

A 66-year-old man with end stage renal disease secondary to hypertension presented for a cadaveric renal transplant. There was no history of prior abdominal surgery.

He received a cadaveric renal transplant via the standard curvilinear Gibson incision to the right lower quadrant. During the procedure an inadvertent $4 \mathrm{~cm}$ peritonotomy was repaired with a running \#3-0 Vicryl suture. The abdominal wall was closed in one layer with figure of eight \#0 Ethibond sutures. Immunosuppression included Almetuzumab induction followed by Mycophenolate and Tacolimus, without steroids.

Postoperatively there was delayed graft function but the patient was eventually discharged home on Day seven. Four days after discharge he returned to the emergency department with nausea, vomiting, obstipation, crampy abdominal pain, and mild distention with tenderness to palpation at the right lower quadrant. An abdominal CT scan showed a high grade small bowel obstruction (Figure 4).

We suspected a hernia through a peritoneal defect and elected to pursue a diagnostic laparoscopy which confirmed our suspicion. The hernia was repaired by opening the peritoneal defect widely and reducing the bowel back into the abdominal cavity. There was no evidence of bowel ischemia and the fascial closure remained intact.

Bowel function resumed on postoperative day two and the patient was discharged home the following day.

\section{Discussion}

Internal hernias are uncommon, accounting for less than $1 \%$ of all intestinal hernias. Paraduodenalhernia have been more commonly described in liver transplantation [3] [4].

Kyriakides et al. first described renal paratransplant hernia in 1978. In their series they reported three cases which presented with bowel obstruction following kidney transplantation. All cases were initially managed nonoperatively but ultimately required exploratory laparatomy. One case required bowel resection and one needed omental resection. The peritoneal defect was repaired in all cases.

P. Cascales-Sanchez et al. also reported thee cases of renal paratransplant hernias [5]. All patients presented with bowel obstruction. The diagnosis was established with abdominal CT scan. One patient needed resection of $20 \mathrm{~cm}$ of small bowel. In two cases the peritoneal defect was closed primarily and in one they used polypropylene prosthesis.

Gao et al. reported their experience with three cases of renal paratransplant hernias [2]. One patient exhibited abdominal pain, nausea, vomiting and distention. After failing nonoperative management for several days an exploratory laparotomy was performed with small bowel resection. The patient developed sepsis and died of multiple organ failure one week following laparotomy. The other two patients survived but had prolonged hospital stays. 


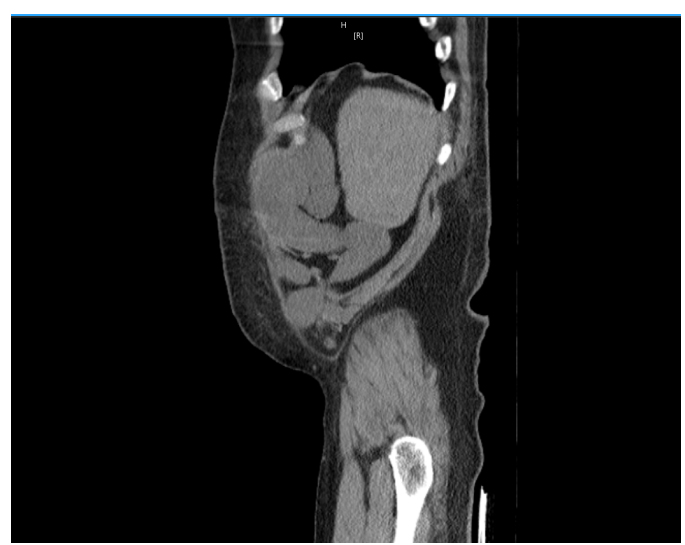

Figure 4. CT scan demonstrating small bowel obstruction with transition point through peritoneal defect.

All reported cases occurred within the first few weeks following transplantation. Historically, exploratory laparotomy has been the standard approach for patients with bowel obstruction. However, re-exploration of a recent transplant incision can increase the risk of wound complications and including surgical site infection and incisional hernia which may require the use of synthetic mesh or Vacuum-assisted closure therapy [6] [7]. A laparoscopic approach minimizes these potential complications.

To our knowledge our report is the first in laparoscopic management of paratransplant hernia. Early diagnosis with CT scan and rapid intervention was the key in our successful result.

In renal transplantation, when meticulous surgical technique fails to prevent perioperative incisional hernia, laparoscopic management can provide definitive treatment in selected cases with minimal risk of further wound complication.

\section{References}

[1] Kyriakides, G.K., et al. (1978) "Paratransplant” Hernia: Three Patients with a New Variant of Internal Hernia. The American Journal of Surgery, 136, 629-630. http://dx.doi.org/10.1016/0002-9610(78)90323-9

[2] Gao, Z.L., et al. (2011) Renal Paratransplant Hernia: A Surgical Complication of Kidney Transplantation. Langenbeck's Archives of Surgery, 396, 403-406.

[3] McIver, M.A. (1933) Acute Intestinal Obstruction. The American Journal of Surgery, 19, 163. http://dx.doi.org/10.1016/S0002-9610(33)90498-5

[4] Khanna, A., et al. (1997) Internal Hernia and Volvulus of the Small Bowel Following Liver Transplantation. Transplant International, 10, 133-136.

[5] Cascales-Sanchez, P., et al. (2007) Renal Paratransplant Hernia: An Unusual Complication of Renal Transplantation? Transplantation Proceedings, 39.

[6] Mazzucchi, E., et al. (2001) Incisional Hernia and Its Repair with Polypropylene Mesh in Renal Transplant Recipients. The Journal of Urology, 166, 816-819. http://dx.doi.org/10.1016/S0022-5347(05)65843-0

[7] Shrestha, B.M., et al. (2007) Vacuum-Assisted Closure (VAC) Therapy in the Management of Wound Infection Following Renal Transplantation. 
Scientific Research Publishing (SCIRP) is one of the largest Open Access journal publishers. It is currently publishing more than 200 open access, online, peer-reviewed journals covering a wide range of academic disciplines. SCIRP serves the worldwide academic communities and contributes to the progress and application of science with its publication.

Other selected journals from SCIRP are listed as below. Submit your manuscript to us via either submit@scirp.org or Online Submission Portal.
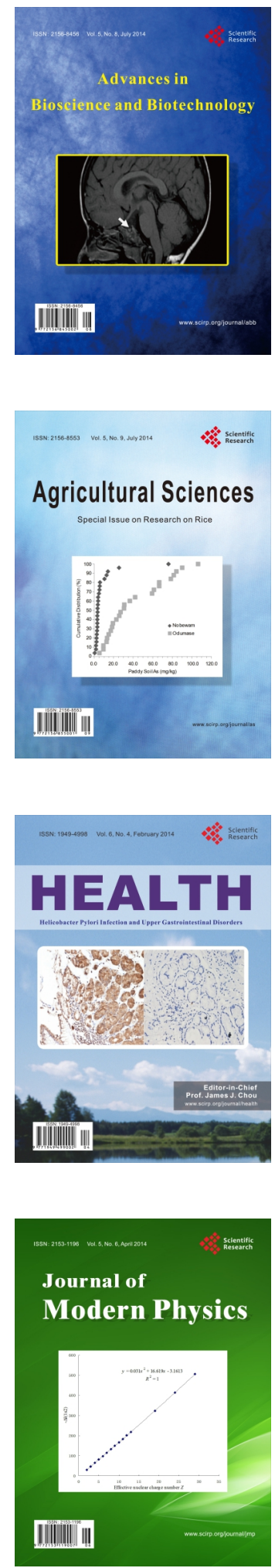
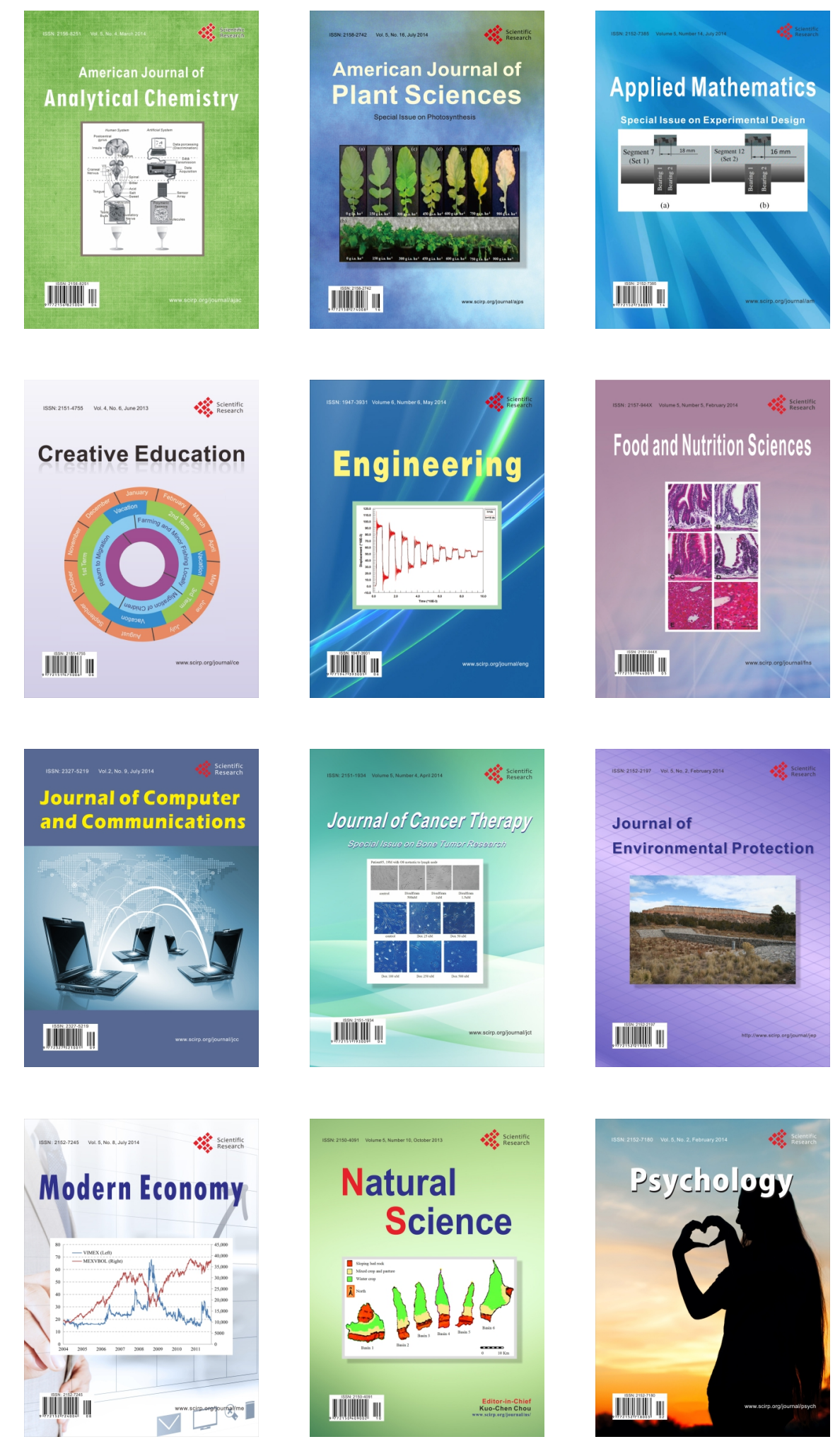